\title{
Introducción a los Sistemas de Gestión de la Calidad Total: Modelo de Excelencia EFQM y Autoevaluación.
}

\author{
Introduction to Total Quality Management Systems: EFQM Excellence Model \\ and self assessment.
}

\author{
Alfredo Robledillo Colmenares ', Daniel Velázquez López ' \\ 1. Colaboradores en la Escuela Nacional de Medicina del Trabajo (Instituto de Salud Carlos III).Madrid. España. \\ Recibido: 29-05-13 \\ Aceptado: 02-08-13
}

\section{Correspondencia}

Alfredo Robledillo Colmenares

Teléfono: +34 680796753

Correo electrónico: alfredorc.danielv|@gmail.com

Resumen

La alta competencia existente entre las organizaciones, acentuada por la vigente situación económica, hace necesaria la utilización de diversas herramientas de gestión de la calidad para mantener y mejorar su eficacia a lo largo del tiempo.

Siendo dicha gestión imprescindible en todos los sectores empresariales, cabe esperar que esta adquiera una mayor relevancia en el ámbito sanitario dado que el principal objetivo que se plantea desde las instituciones de la salud es obtener la satisfacción de los pacientes y de la sociedad en general.

Dentro de las herramientas para la mejora continua de la calidad, una de las más utilizadas es la autoevaluación, que consiste en un examen de la propia organización en comparación con un modelo de perfección. De esta manera las empresas pueden identificar sus puntos fuertes y sus áreas de mejora y, por ende, conocer el punto en el que se encuentran respecto a la excelencia.

De entre los modelos de perfección existentes, el Modelo propuesto desde la Fundación Europea de Gestión de la Calidad, el Modelo EFQM, es el que presenta un uso más extendido en la actualidad en Europa.

En las siguientes páginas se presenta de una manera sencilla la estructura y los conceptos fundamentales tanto del procedimiento de autoevaluación como del Modelo EFQM, todo ello con el propósito de acercar este tipo de herramientas a los profesionales de la salud y proporcionar a los mismos nociones básicas acerca de su implantación.

Med Segur Trab (Internet) 2013; 59 (232) 302-309

Palabras clave: Modelo de Excelencia EFQM, autoevaluación, Gestión de la Calidad Total, mejora continua.

Abstract

The high competence existing between organisations, increased by the current economic situation, requires the use of various quality management tools to mantain and improve their efectiveness over time.

This management is essential in every business sector, but is expected to be more relevant in the health area as the main aim in this field is achieving patients and society satisfaction. 
Among the tools for continuous quality improvement, self assessment is one of the most used, which is a review of the own organisation compared with a perfection model. This procedure enables organisations to identify their strengths and improvement areas and therefore to know their current situation in relation with quality.

Nowadays the model proposed by the European Foundation for Quality Management, called EFQM Excellence Model, is the most extendedly used all over Europe.

The following pages present in a simple manner the structure and the fundamental concepts of the self assessment procedure and the EFQM Model, all of it with the main objective of making health professionals aware about these kind of tools and providing them the basic ideas about its implementation.

Med Segur Trab (Internet) 2013; 59 (232) 302-309

Key words: EFQM Excellence Model, self assessment, Total Quality Management, continuous improvement. 


\section{ANTECEDENTES HISTÓRICOS}

Aunque habitualmente se asocia el concepto de calidad al S. XX, siempre ha existido un concepto intuitivo de la misma para el ser humano dada su preocupación innata por los trabajos bien hechos.

Las raíces de los modelos de calidad se remontan a la Europa de finales del Siglo XIII, cuando los artesanos comenzaron a organizarse en gremios elaborando normas estrictas orientadas a mejorar la calidad de sus productos y servicios. Será siglos después, con el comienzo de la Revolución Industrial de 1800, cuando aparezcan modelos más estructurados de control de la producción.

A partir del año 1900 la evolución del concepto fue muy dinámica, pues se adaptó al progreso de la industria, y de forma paralela se desarrollaron diversas teorías, fundamentos y técnicas hasta llegar a lo que actualmente se conoce como Calidad Total.

La Segunda Guerra Mundial jugó un papel decisivo en el desarrollo de los sistemas de control de calidad, ya que los países que intervinieron en la misma trataban de garantizar un rendimiento eficaz de sus materiales bélicos.

Al finalizar la guerra, Japón, como la mayoría de las naciones que intervinieron en ella, emprende una reconversión de las infraestructuras destinadas a producción de materiales militares en dependencias orientadas hacia la producción de material de uso civil, tanto para alimentar las demandas internas como para atender a las del comercio exterior.

La baja calidad de los productos en sus primeros intentos por incorporarse a este mercado llevó a este país a adquirir una mala reputación de su cadena de producción con el consiguiente rechazo de los mercados internacionales. La primera estrategia adoptada por Japón para contrarrestar este efecto negativo fue la apertura de sus espacios industriales a empresas extranjeras, lo que propició la llegada de W. Edwards Deming, quien había trabajado en EEUU en los procesos de control de calidad durante el periodo de producción bélica.

De esta forma se dio un paso más avanzado en concepto de sistemas de control de calidad, orientándolo hacia la idea anteriormente mencionada de Calidad Total, donde lo que se revisa es cada uno de los procesos que intervienen en el procedimiento de fabricación, involucrando a las personas que intervienen durante los mismos y que los utilizan como herramientas para alcanzar el producto final. El resultado fue una producción de elevada calidad a precios más bajos y competitivos que los competidores y, por tanto, la incorporación masiva de sus productos en los mercados internacionales.

Este nuevo escenario dio lugar a un perjuicio en los intereses de mercado de los EEUU y a una crisis en la calidad de sus productos durante la década de los 70, situación que se subsanó a partir de los 80 cuando se empiezan a establecer estrategias de control de Calidad Total mediante programas diseñados para actuar frente a toda la organización empresarial, y es lo que hoy conocemos como Sistemas de Gestión de Calidad Total o Total Quality Management (TQM).

\section{EL MODELO EFQM}

\subsection{Autoevaluación y modelo EFQM}

La situación económica actual conlleva a las empresas la imperiosa necesidad de mejorar tanto la gestión de sus actividades como sus resultados. En este sentido, en el ámbito empresarial se habla de alcanzar la excelencia, o lo que es lo mismo, llegar al estado de mayor calidad posible mediante la aplicación de diversos procedimientos y técnicas profesionales, aumentando de esta manera la satisfacción de los clientes y mejorando la gestión de los recursos y de los procesos. 
La forma de gestión más novedosa para tratar de alcanzar dicho estado de perfección es la aplicación del concepto de Calidad Total, que, como se ha explicado anteriormente, consiste no solo en la revisión del resultado final de las actividades de la organización, sino también en el seguimiento de todos los procesos intermedios utilizados para obtener dicho resultado, con la finalidad de minimizar en la medida de lo posible los errores que puedan surgir durante la fabricación de los productos.

La gestión de una organización a través de un Sistema de Calidad Total requiere conocer previamente los niveles vigentes de calidad de la gestión y de los resultados en una empresa; una de las herramientas más completas para obtener este conocimiento es la realización de una autoevaluación.

Una autoevaluación consiste en un examen global, sistemático y regular de las actividades y resultados de una organización comparados con un modelo de excelencia empresarial; dicho de otra manera, se trata de un procedimiento mediante el cual las empresas se comparan con un modelo de perfección para determinar el punto en el que se encuentran con respecto a la excelencia, identificando tanto sus puntos fuertes como sus áreas susceptibles de mejora. De esta manera, los resultados obtenidos al aplicar esta herramienta pueden utilizarse como punto de partida para planificar la actividad empresarial y para establecer planes de mejora.

De entre los diversos modelos existentes para llevar a cabo esta comparación, el más utilizado en los últimos años ha sido el Modelo Europeo de Excelencia Empresarial propuesto por la Fundación Europea para la Gestión de la Calidad, cuyas siglas en inglés son EFQM, una entidad sin ánimo de lucro que alberga como misión principal fomentar la excelencia en las organizaciones europeas de una manera sostenida.

Se trata de un modelo no prescriptivo, es decir, a diferencia de otros instrumentos de gestión de la calidad, no se encuentra sujeto a ninguna normativa, lo cual le otorga un notable carácter de flexibilidad y adaptabilidad y hace que pueda ser aplicable a cualquier tipo y tamaño de empresa.

Igualmente, se trata de una herramienta permanentemente actualizada y que se encuentra validada por el uso y experiencia previa de numerosas organizaciones.

\subsection{Estructura del modelo EFQM}

El Modelo de Excelencia EFQM está basado en una serie de criterios, los cuales constituyen el objeto de comparación durante la autoevaluación. Su cuidada y rigurosa elaboración por parte de la EFQM hace que los mismos sean ampliamente aceptados en Europa.

El Modelo contempla nueve criterios que se pueden diferenciar en dos grupos:

- Cinco de ellos son los denominados agentes facilitadores, es decir, lo que la organización hace:

- Liderazgo: encuadra las acciones que lleva a cabo la Alta Dirección para promover la cultura de la Calidad Total.

- Política y estrategia: hace referencia a como la organización elabora y desarrolla su plan estratégico.

- Personas: refleja la forma en que una empresa aprovecha el potencial de sus empleados.

- Alianzas y recursos: como gestiona la organización sus alianzas externas y sus recursos internos.

- Procesos: engloba no solo la manera en que se diseñan y gestionan los procesos, si no como se trata de mejorarlos.

- Los cuatro restantes se incluyen dentro de los resultados, o lo que es lo mismo, lo que la organización logra:

- Resultados en los clientes. 
- Resultados en las personas.

- Resultados en la sociedad.

- Resultados clave o logros alcanzados en relación al rendimiento planificado.

Cabe mencionar que cada criterio se estructura en un número variable de subcriterios, existiendo un total de treinta y dos.

En aras de la mejora continua de la gestión de la calidad, el proceso de evaluación de cada criterio por parte de las organizaciones sigue un esquema lógico denominado REDER:

- En primer lugar, se han de determinar los RESULTADOS a lograr en relación a cada uno de los criterios.

- A continuación, se planifican y desarrollan los ENFOQUES necesarios para alcanzar dichos resultados.

- Una vez planificados, se efectúa el DESPLIEGUE de los enfoques.

- Por último, se lleva a cabo la EVALUACIÓN y REVISIÓN de los enfoques y su despliegue.

La aplicación de este ciclo de evaluación de una manera repetida permite a las empresas alcanzar una mejora de su excelencia de una manera sostenida.

\subsection{Metodología de puntuación del modelo EFQM}

El Modelo de Excelencia de la EFQM, además de permitir la identificación de puntos fuertes y áreas de mejora, incluye una metodología objetiva que permite obtener una puntuación numérica.

La puntuación total de una organización será el resultado de la suma de las puntuaciones obtenidas en cada uno de los criterios que componen el Modelo. Cada uno de ellos cuenta con un peso específico, tal y como queda reflejado en la Figura 1, pudiendo obtenerse un máximo de 1.000 puntos, de los cuales la mitad corresponden a los agentes facilitadores y la otra mitad a los resultados.

Figura 1. Mapa de Criterios

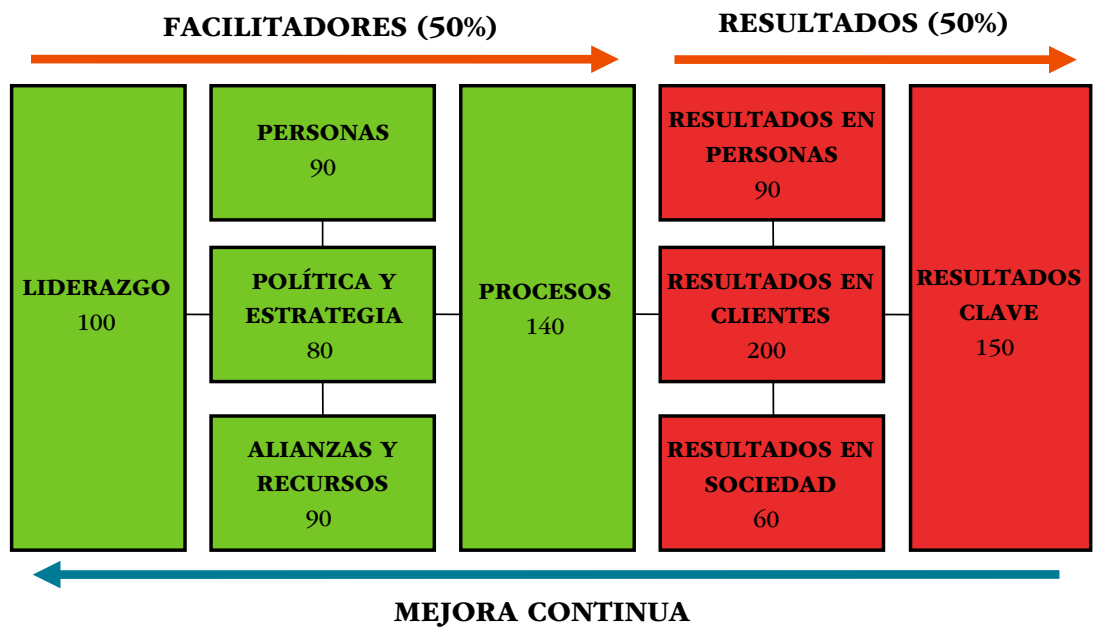

En consecuencia, este sistema permite a las organizaciones calcular resultados cuantitativos de sus autoevaluaciones, pudiendo comparar los mismos con los obtenidos en autoevaluaciones realizadas con carácter previo; es decir, constituye un indicador de seguimiento de la eficacia de los proyectos de mejora adoptados anteriormente.

Asimismo, este sistema de cuantificación supone una herramienta que permite a las organizaciones comparar de una manera objetiva su nivel de excelencia frente a otras empresas, técnica que se conoce en el mundo de los negocios como benchmarking. 
Además, dado que el Modelo puede aplicarse o bien a la totalidad de la organización o bien únicamente a una parte de esta, se podrían contrastar los valores numéricos obtenidos en distintas unidades o centros de una misma entidad.

Cabe reseñar que las puntuaciones obtenidas constituyen el fundamento para la concesión del Premio Europeo a la Calidad, creado y gestionado por la propia Fundación. Todas las entidades que implanten el Modelo pueden optar voluntariamente a este reconocimiento, obteniendo un nivel de excelencia de $+200,+300,+400$ o +500 en función de las actividades de gestión llevadas a cabo y de los resultados conseguidos.

\subsection{Enfoques de autoevaluación del modelo EFQM}

Como se ha indicado anteriormente, el Modelo EFQM se caracteriza por su adaptabilidad, afirmación que se fundamenta en el hecho de que la Fundación no contempla una única metodología para desarrollar una autoevaluación si no que desde la misma se proponen hasta cinco posibles enfoques para llevarla a cabo.

Cada uno de estos enfoques establece una metodología distinta para recopilar la información relativa a los puntos fuertes y a las áreas de mejora existentes en la organización; todo ello deberá quedar apoyado en evidencias, es decir, documentación que avale los hallazgos.

La elección de un método u otro dependerá tanto de los objetivos que se persigan con la autoevaluación como de las particularidades de cada empresa: cultura organizacional, experiencia en gestión de la Calidad Total, disponibilidad de tiempo, etc.

A continuación se exponen de manera sintética cada uno de los cinco enfoques propuestos desde la EFQM:

Autoevaluación por Simulación de Presentación al Premio: consiste en la elaboración de una memoria completa en la cual se desarrollará un capítulo por cada criterio del Modelo, especificando las acciones llevadas a cabo en cada uno de ellos.

Autoevaluación por Formularios: conlleva la elaboración de treinta y dos formularios, uno por cada subcriterio en los que se encuentra estructurado el Modelo.

Autoevaluación por Matriz de Mejora: en este enfoque se utiliza una matriz compuesta por nueve columnas, que se corresponden con los criterios del Modelo, y diez filas, cada una de las cuales refleja el grado de consecución de los criterios.

Autoevaluación por Reunión de Trabajo: supone la asignación de los subcriterios a distintos miembros de la dirección de la organización, quienes se encargarán de recopilar información relativa a los mismos. Posteriormente, en una reunión de trabajo, se ponen en común los datos recabados y se adoptan decisiones de una forma consensuada.

Autoevaluación por Cuestionario de Autoevaluación: requiere el uso de cuestionarios específicos para cada criterio que serán planteados a distintos miembros de la empresa.

Cada uno de los enfoques requerirá un nivel distinto de esfuerzo y de madurez en la excelencia; por ejemplo, la elaboración de una memoria supone un trabajo mucho más arduo del que supondría distribuir cuestionarios entre los empleados así como una mayor experiencia en la gestión de la calidad. Por tanto, la variedad de metodologías para desarrollar el procedimiento otorga a las organizaciones la posibilidad de elegir el enfoque más adecuado a sus necesidades y a sus condiciones particulares.

\subsection{Proceso general de autoevaluación}

Independientemente del método seleccionado por cada organización, el proceso general de autoevaluación es siempre el mismo.

Previamente al comienzo del procedimiento, es fundamental asegurar que la dirección de la organización se encuentra sólidamente comprometida con la mejora 
continua y que existe una aceptación del Modelo EFQM. En este sentido, suele ser necesaria la impartición de formación inicial centrada en estos aspectos.

La autoevaluación propiamente dicha comienza con la planificación de la misma. Esta incluye el establecimiento de los objetivos perseguidos, la designación de tareas, el diseño del calendario de acciones y la determinación de los recursos humanos y materiales necesarios para el proceso. Asimismo, en esta etapa se seleccionará el enfoque más adecuado, de entre los cinco propuestos por la EFQM, para desarrollar la autoevaluación.

Posteriormente, habrán de constituirse los equipos encargados de desarrollar el procedimiento, impartiendo a los integrantes la formación oportuna. En este punto, es imprescindible cerciorarse de que los objetivos que persigue la autoevaluación quedan perfectamente clarificados.

Tras la planificación y la constitución de los equipos se llevará a cabo la ejecución del enfoque elegido. Es importante señalar que la autoevaluación conlleva la generación de ideas por parte de los miembros de los equipos y, lo que es más significativo, la adopción de decisiones basadas en el consenso.

Los resultados obtenidos tras la aplicación del enfoque constituirán el punto de partida a partir del cual la empresa establecerá un plan de acción enfocado, por un lado, en potenciar los puntos fuertes identificados y, por otro, en tratar las áreas susceptibles de ser mejoradas. A la hora de diseñar los planes de acción o planes de mejora se han de incluir las líneas de acción a seguir, ordenadas según su prioridad, los responsables, el calendario, los recursos necesarios y las medidas de seguimiento y control del progreso de las acciones. Una posible manera de examinar dicho progreso consistiría en el desarrollo de nuevas autoevaluaciones que reflejasen la evolución de las puntuaciones obtenidas.

\subsection{Beneficios y dificultades del modelo EFQM}

La aplicación del Modelo EFQM en cualquier organización supone para la misma una serie de ventajas significativas.

En primer lugar, la correcta utilización de esta herramienta permite a las organizaciones prestar un servicio de alta calidad y aumentar su rentabilidad, lo que en términos generales se traduce en un incremento de la competitividad.

Por otro lado, su implantación requiere de la participación de todos los empleados, de tal manera que al involucrarse en el proyecto se logra un alto grado de motivación a la hora de alcanzar los objetivos y se propicia un medio de formación del personal en el campo de la Calidad Total.

Asimismo, las iniciativas y planes de mejora de la calidad resultantes del procedimiento quedan integradas en el día a día de los trabajadores de la empresa, asegurándose de esta manera su completa y óptima adopción.

Por último, cabe destacar que se trata de un sistema perfectamente compatible con otras herramientas. No supone una contraposición a otros enfoques de control de Calidad Total, sino más bien la integración de los mismos en un esquema más amplio y completo de gestión.

No obstante, con vistas al adecuado establecimiento del sistema, resulta imprescindible tener siempre presentes una serie de factores inherentes al mismo.

Como ocurre con la mayor parte de las técnicas de calidad, se trata de un Modelo que permite obtener resultados a medio y largo plazo. Por tanto, si su implantación se orienta hacia un intento de obtener beneficios rápidamente, aparecerán normalmente problemas como la precipitación o la inconsistencia del trabajo realizado.

Igualmente importante para el éxito del procedimiento es su planificación, sobre todo en lo que se refiere a los recursos y a la formación. En caso de que dicha planificación 
sea inadecuada, es frecuente que se originen problemas de inseguridad y rechazo del proyecto.

Finalmente, resulta interesante reseñar un inconveniente muy común en las herramientas modernas de calidad: el rechazo que estas generan en el personal de las organizaciones. La falta de aceptación de estos métodos suele deberse a dos factores; primeramente, la falsa creencia de que su uso supondrá un exceso de burocracia y una sobrecarga de trabajo; por otro lado, la frecuente actitud de resistencia al cambio, es decir, el temor a lo nuevo. Ante estas percepciones, se habrán de utilizar la sensibilización, comunicación, formación e información como medios para contrarrestarlas.

\section{APLICACIONES DEL MODELO EFQM}

A día de hoy, el Modelo EFQM se encuentra implantado exitosamente en una gran cantidad de organizaciones a lo largo de toda Europa.

De una manera más concreta, su aplicación a nivel nacional ha tenido lugar en numerosas empresas, principalmente en todas aquellas en las que concurren procesos bien definidos, como es el caso de las bibliotecas. Asimismo, ha sido utilizado en múltiples entidades del sector sanitario, como hospitales o centros de investigación.

Sirva a modo de ejemplo de aplicación concreta de esta herramienta el caso de la Red Europea de Promoción de la Salud en el Trabajo, cuyas siglas en inglés son ENWHP. Este organismo se encargó de llevar a cabo una adaptación del Modelo, de tal manera que a través de una configuración distinta de los criterios del mismo se consiguió que fuese aplicable al campo de la Promoción de la Salud.

\section{REFERENCIAS BIBLIOGRÁFICAS}

1. Basic Concepts. The History of Quality http://asq.org/learn-about-quality/history-of-quality/overview/overview.html (consultado el 12 de junio de 2013)

2. EFQM - Introducción a la Excelencia. Universidad Católica de Valencia. https://www.ucv.es/documentos/calidad/EFQM.pdf (consultado el 20 de junio de 2013)

3. Modelo de Excelencia EFQM de ADER (Agencia Desarrollo Económico de La Rioja) http://www.arnaut.net/sites/default/files/images/documentos/ader-modelo-efqm.pdf (consultado el 20 de junio de 2013)

4. Presentación del Modelo EFQM. Guía de la Calidad http://www.guiadelacalidad.com/files/pdf/Presentacion_del_modelo_EFQM.pdf (consultado el 20 de junio de 2013)

5. Certificación bajo el Modelo EFQM de Excelencia Europea. Asociación Española de Normalización y Certificación (AENOR)

http://www.aenor.es/aenor/certificacion/calidad/calidad_efqm.asp\#.UbhN1Ngm60Q (consultado el 20 de junio de 2013)

6. European Foundation for Quality Management www.efqm.org (consultado el 20 de junio de 2013)

7. Membrado Martínez, J. La gestión empresarial a través del modelo europeo de excelencia de la EFQM. Díaz de Santos, SA. 1999.

8. Martín Castilla, J.I. EFQM - Administración. En busca de la Excelencia al servicio del ciudadano de la Comunidad de Madrid. Dirección General de Servicios y Atención al Ciudadano. 2007. $2^{\mathrm{a}}$ ed. Madrid. España.

| 